\title{
Complementary roles of IRS-1 and IRS-2 in the hepatic regulation of metabolism
}

\author{
Cullen M. Taniguchi, ${ }^{1}$ Kohjiro Ueki, ${ }^{1,2}$ and C. Ronald Kahn'1 \\ ${ }^{1}$ Research Division, Joslin Diabetes Center, Harvard Medical School, Boston, Massachusetts, USA. \\ 2Department of Metabolic Diseases, Graduate School of Medicine, The University of Tokyo, Tokyo, Japan.
}

\begin{abstract}
Hepatic insulin resistance is a critical component in the development of type 2 diabetes mellitus. In many cases, insulin resistance in liver is associated with reduced expression of both major insulin receptor substrate (IRS) proteins, IRS-1 and IRS-2. To investigate the specific functions of IRS-1 and IRS-2 in regulating liver function in vivo, we developed an adenovirus-mediated RNA interference technique in which short hairpin RNAs (shRNAs) are used to knock down IRS-1, IRS-2, or both, by 70-80\% in livers of WT mice. The knockdown of IRS-1 resulted in an upregulation of the gluconeogenic enzymes glucose- 6 phosphatase and phosphoenolpyruvate carboxykinase, as well as a marked increase in hepatic nuclear factor-4 $\alpha$. Decreased IRS- 1 was also associated with a decrease in glucokinase expression and a trend toward increased blood glucose, whereas knockdown of IRS-2 resulted in the upregulation of lipogenic enzymes SREBP-1c and fatty acid synthase, as well as increased hepatic lipid accumulation. The concomitant injection of IRS-1 and IRS-2 adenoviral shRNAs resulted in systemic insulin resistance, glucose intolerance, and hepatic steatosis. The alterations in the dual-knockdown mice were associated with defective Akt activation and Foxo1 phosphorylation. Taken together, our results demonstrate that hepatic IRS-1 and IRS-2 have complementary roles in the control of hepatic metabolism, with IRS-1 more closely linked to glucose homeostasis and IRS-2 more closely linked to lipid metabolism.
\end{abstract}

\section{Introduction}

Insulin resistance in liver contributes greatly to the development of type 2 diabetes mellitus (1-3). Decreased hepatic insulin sensitivity leads to postprandial hyperglycemia and increased hepatic glucose production, which exacerbates an already deleterious situation of hyperglycemia and chronic hyperinsulinemia in diabetics (4). In addition to affecting glucose levels, hepatic insulin resistance may also lead to dysregulated lipid synthesis, which can lead to hepatic steatosis and further systemic insulin resistance (5).

Hepatic insulin resistance may stem from compromised signaling through the insulin receptor substrate (IRS) proteins, a family of docking molecules that connect insulin receptor activation to essential downstream kinase cascades, such as the PI3K or MAPK pathways. The 2 major IRS isoforms, IRS-1 and IRS-2, are highly expressed in livers of normal mice but are downregulated to various extents in livers of diabetic animals and humans (6-10). Since IRS proteins are a critical link in hepatic insulin signaling, it has been hypothesized that the decreased expression of IRS proteins in liver may be a key molecular lesion of hepatic insulin resistance $(5,11)$.

Despite many physiologic and molecular studies, it is still unclear whether the downregulation of IRS proteins is causative,

Nonstandard abbreviations used: Abca1, ATP-binding cassette, subfamily A1; Acadm, medium chain acyl-CoA dehydrogenase; Cpt1a, carnitine palmitoyl transferase-1; Cyp7a1, cytochrome P450, isoform 7A1; F-1,6BP, fructose-1,6-bisphosphatase; Fasn, fatty acid synthase; G6Pase, glucose-6 phosphatase; Gck, glucokinase; GTT, glucose tolerance test(ing); HNF- $4 \alpha$, hepatic nuclear factor- $4 \alpha$; IRS, insulin receptor substrate; IRS1U6, adenovirus expressing shRNAs against IRS-1; ITT, insulin tolerance test; LXR, liver X receptor; MODY, maturity onset diabetes of the young; PEPCK, phosphoenolpyruvate carboxykinase; PY, phosphotyrosine; RNAi, RNA interference; shRNA, short hairpin RNA; siGFP, adenovirus expressing shRNAs against GFP; TG, triglyceride.

Conflict of interest: The authors have declared that no conflict of interest exists.

Citation for this article: J. Clin. Invest. 115:718-727 (2005)

doi:10.1172/JCI200523187. or merely correlative, with pathophysiology and whether IRS-1 or IRS-2 play unique roles in hepatic insulin action. Individual global knockouts of IRS-1 (12) and IRS-2 (13) do not exhibit an obvious hepatic phenotype, and IRS-1/IRS-2 KO mice die in utero (14). The lack of a liver phenotype in the individual-knockout animals is partially explained at the molecular level, since IRS-1-KO mice have no defects in hepatic phosphotyrosine-associated (PY-associated) PI3K activity because a constitutive increase in IRS-2 protein levels is able to fully compensate for defects in IRS-1 expression (15). On the other hand, the restoration of IRS-1 expression in the livers of IRS-1-KO mice is sufficient to completely normalize insulin sensitivity, which indicates that hepatic IRS-1 is critical to metabolic homeostasis (16). Euglycemic clamp studies have suggested that IRS-2-KO mice may have a greater defect in the insulin-mediated suppression of hepatic glucose production than IRS-1-KO mice (17), and IRS-2-KO mice exhibit a $50 \%$ decrease in PY-associated PI3K activity in the liver, with no increase in IRS-1 levels (18). These data must be interpreted cautiously, however, since lifelong knockout of either IRS protein could result in pleiotropic changes that might confound the interpretation of the molecular functions of IRS- 1 and IRS-2. For instance, IRS-2-KO mice exhibit central leptin resistance $(19,20)$, a defect that, by itself, may compromise hepatic insulin signaling (21).

To better understand the roles of IRS- 1 and IRS- 2 in hepatic insulin action, we developed an adenovirus-mediated RNA interference (RNAi) technique that utilizes short hairpin RNAs (shRNAs) to substantially and stably knock down IRS-1 and IRS-2 expression specifically in the livers of WT mice. While RNAi has been an effective tool for in vitro studies of gene function, its in vivo use has been limited. Transgenic (22) and lentiviral (23) shRNA systems have proven to be effective in abrogating gene expression in all tissues in mice, but only recently have techniques been developed to use RNAi in a tissue-specific manner (24-26). 
A

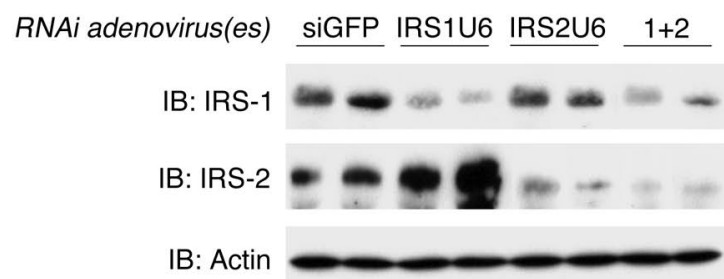

B IB: IRS-1

Adenovirus

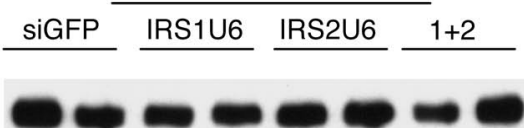

Muscle

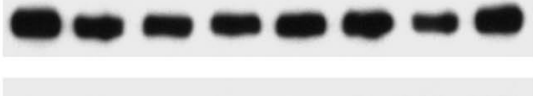

Fat

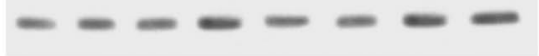

Kidney

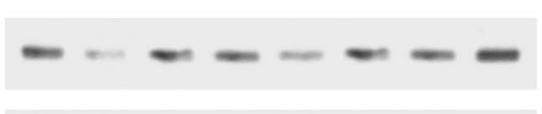

Brain

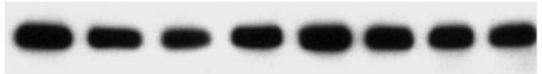

C
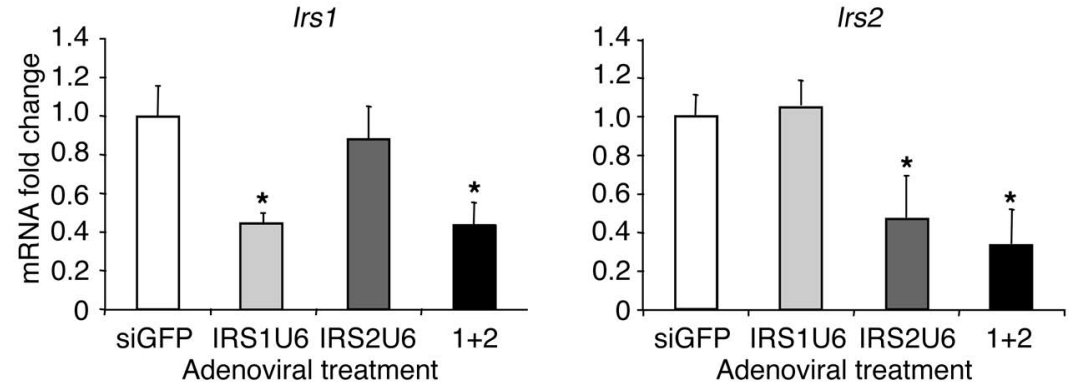

D

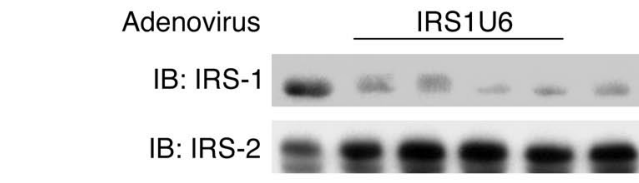

Days after injection:
13
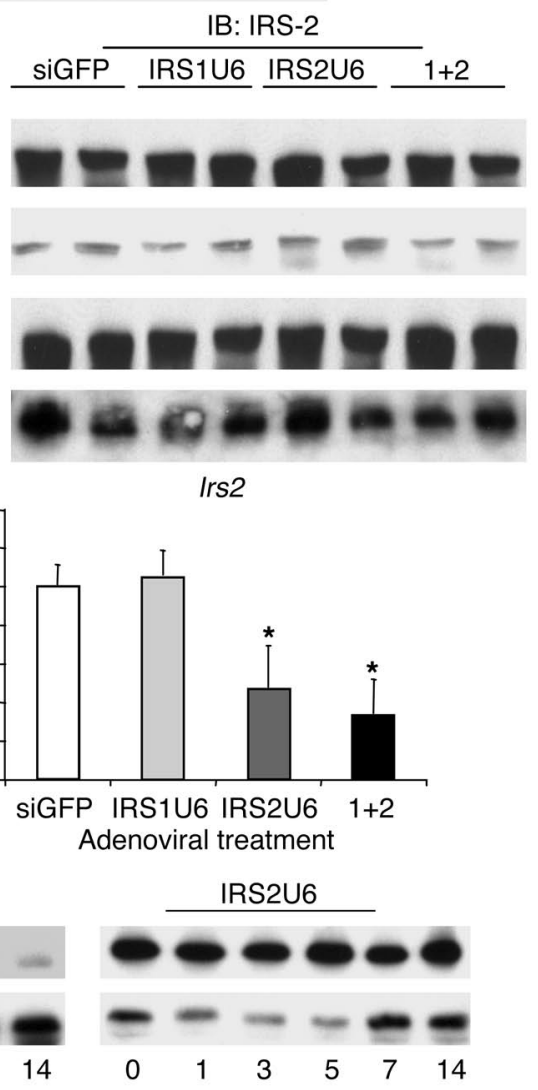

Figure 1

RNAi adenoviruses cause substantial and specific knockdown of IRS-1 and IRS-2 in liver. (A) Western blots for IRS-1, IRS-2, and actin in liver lysates from mice treated with siGFP, IRS1U6, IRS2U6, or both IRS1U6 and IRS2U6 (1+2). Livers were collected from mice after an overnight fast, and proteins were extracted and processed as described in Methods. Each lane represents liver lysates from a different mouse. (B) Muscle, fat, kidney, and brain lysates of the mice represented in $\mathbf{A}$ were subjected to immunoblots for IRS-1 and IRS-2. (C) Quantitative RT-PCR analysis of IRS-1 and IRS-2 mRNA levels in livers 5 days after treatment with siGFP, IRS1U6, IRS2U6, or both viruses simultaneously $(n=6)$. Total RNA was extracted from livers and made into CDNA, and primers specific to IRS-1 and IRS-2 were used to determine levels of expression, as described previously (58). (D) Time course of effect of RNAi adenovirus. Liver lysates were made from mice 1 , $3,5,7$, or 14 days after viral injection and blotted for IRS-1 and IRS-2 proteins levels. Day 0 liver lysates were obtained from age-matched mice injected with saline. These results are representative of 3 independent experiments.
In the present study, we have taken advantage of the relative tissue specificity of adenovirus for liver and the genetic specificity of shRNA-mediated RNAi to create liver-specific hypomorphs of IRS-1 and IRS-2. By knocking down IRS-1 and IRS-2 separately and together in liver, we show that IRS-1 and IRS-2 work together via mutual compensation to maintain total PI3K activity and also have unique roles in gene regulation. IRS- 1 signaling may be more closely linked to the regulation of genes involved in glucose homeostasis, whereas IRS-2 signaling may have specific roles in the regulation of hepatic lipid metabolism. Moreover, the concomitant knockdown of IRS-1 and IRS-2 in liver results in fasting hyperglycemia, fasting hyperinsulinemia, insulin resistance, glucose intolerance, dyslipidemia, and other characteristics consistent with the metabolic syndrome.

\section{Results}

Adenovirus-mediated RNAi significantly and specifically reduces IRS expression in the liver. Five days after tail-vein injection of an adenovirus expressing shRNAs against IRS-1 (IRS1U6), there was an average $80 \%$ decrease in IRS- 1 protein levels in liver, as compared with mice injected with a control adenovirus that expressed shRNAs against GFP (siGFP; Figure 1A). This knock- down of IRS- 1 was specific. There was no associated decrease in actin expression, and IRS- 2 protein levels actually increased by $20 \%$ in mice treated with IRS1U6. Similarly, recombinant RNAi adenovirus against IRS-2 (IRS2U6) specifically reduced IRS-2 protein expression by approximately $70 \%$, without altering IRS-1 levels or actin controls.

Although others have used this shRNA approach on single genes with success (27), there are no known published studies that use adenoviral shRNAs to knock down multiple genes simultaneously. Consequently, we were concerned that dual knockdown could result in greater nonspecific effects than single viral treatment. We found that concomitant treatment with both IRS1U6 and IRS2U6 caused substantial knockdown of both IRS-1 and IRS-2 at levels similar to single treatments, without inducing any decreases in levels of actin protein. Dual adenoviral treatment also did not cause any nonspecific changes in protein expression of other insulin signaling molecules, such as the insulin receptor $\beta$ subunit, GSK3 $\beta$, and MAPK (data not shown), nor did they cause an appreciable increase in serum levels of the liver enzymes alanine aminotransferase (ALT) and aspartate aminotransferase (AST; see Methods). Moreover, the effects of adenoviral shRNAs were also tissue specific, as there 


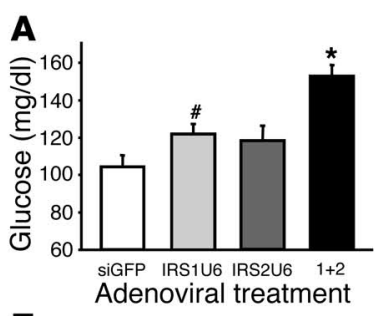

$\mathbf{E}$

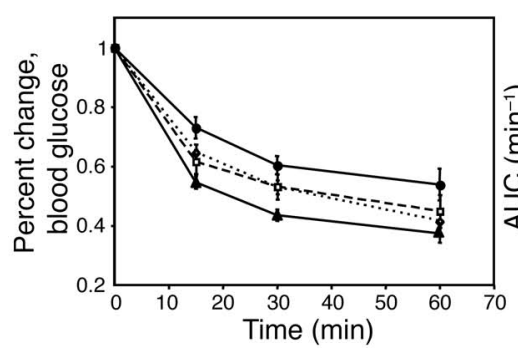

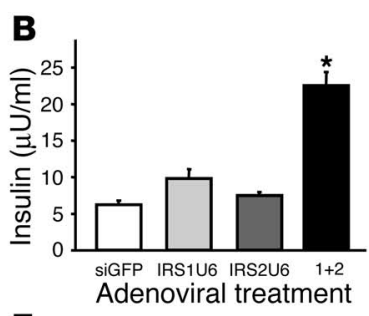

$\mathbf{F}$

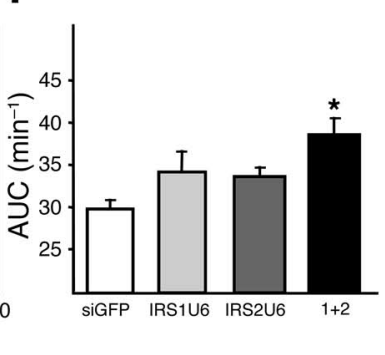

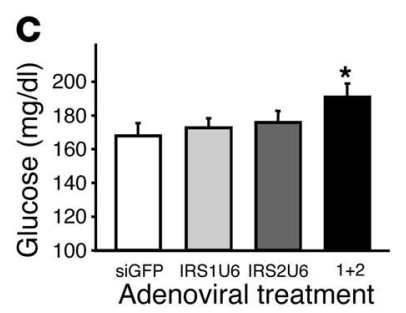

G

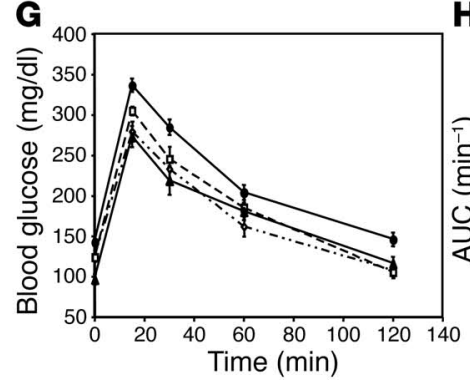

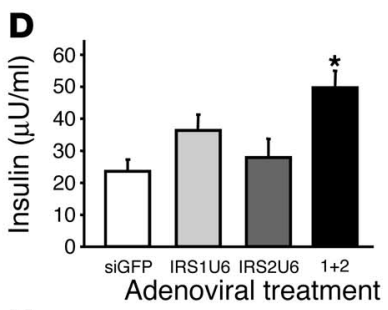

H

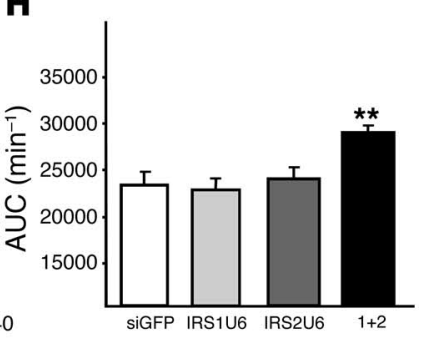

Figure 2

Dual knockdown of IRS-1 and IRS-2 results in defects in glucose homeostasis. Fasting blood glucose (A) and insulin levels (B) as well as random-fed blood glucose (C) and insulin levels (D) of mice treated with RNAi adenovirus. Glucose and insulin levels are plotted as the mean \pm SEM ( $n=10-20$ mice per treatment group in both fasted and fed state). (E) Insulin tolerance tests (1 U/kg, i.p.) were performed 5 days after adenovirus injection. Results represent blood glucose concentration as a percentage of starting value at time point 0 and are expressed as mean \pm SEM $(n=8)$. (F) Results from E expressed as area under ITT curves. AUC, area under the curve. (G) Glucose tolerance tests (2 g/kg, i.p.) were performed on mice following a 16-hour fast 5 days after adenovirus injection. Blood samples were collected and glucose measured at the times indicated. (H) Results shown in G expressed as area under GTT curves. Open circles, siGFP; closed squares, IRS1U6; open diamonds, IRS2U6; closed circles, both IRS1U6 and IRS2U6. ${ }^{*} P<0.05$ vs. siGFP; ${ }^{*} P<0.01$ vs. siGFP; ${ }^{*} P=0.06$ vs. siGFP.

were no observed differences in IRS-1 or IRS-2 protein levels in muscle, fat, kidney, and brain between control mice and mice treated with IRS1U6, IRS2U6, or both (Figure 1B).

RNAi may regulate gene expression by several mechanisms, including the inhibition of translation and by degradation of mRNA $(28,29)$. To confirm that mRNA levels were also decreased, we measured the mRNA expression of IRS- 1 and IRS-2 by quantitative RT-PCR (Figure 1C) 5 days after adenovirus injection. We found that IRS-1 mRNA was decreased by $56 \%$ when IRS1U6 was injected either as a single treatment or along with IRS2U6. Similarly, IRS2U6 caused a 53\% drop in IRS-2 mRNA levels when it was given alone and a $66 \%$ decrease when IRS1U6 was also injected.

Since the effect of adenovirus-mediated gene transfer is transient, we wanted to confirm that the RNAi effect against IRS-1 and IRS-2 would last long enough to allow us to perform subsequent metabolic studies. Interestingly, the IRS1U6 virus caused downregulation of IRS-1 levels for up to 2 weeks, while IRS2U6 decreased IRS-2 levels for a more modest 7 days (Figure 1D).

Glucose homeostasis is impaired by the liver-specific downregulation of IRS-1 and IRS-2. To investigate how liver-specific defects in IRS-1 and IRS- 2 affect glucose homeostasis, we measured blood glucose and serum insulin levels in the fasting and random-fed states. Single treatments with IRS1U6 or IRS2U6 did not cause any statistically significant changes in blood glucose levels in fasting or fed mice (Figure 2, A and C), but dual knockdown of IRS- 1 and IRS- 2 caused hyperglycemia in both states. The serum insulin levels in the RNAi adenovirus-treated mice paralleled the changes in blood glucose, as mice treated concomitantly with IRS1U6 and IRS2U6 exhibited hyperinsulinemia in both the fasting and fed states. While there was no difference in insulin levels between mice treated with IRS2U6 and controls (Figure 2, B and D), the levels trended higher in IRS1U6-treated mice compared with controls, although the difference was not statistically significant.

The simultaneous knockdown of IRS- 1 and IRS-2 also resulted in insulin resistance and glucose intolerance. Insulin tolerance tests (ITTs) of mice treated with either IRS1U6 or IRS2U6 detected only mild insulin resistance that did not reach statistical significance (Figure 2E). Double knockdown, however, caused a measurable level of insulin resistance that was statistically different from that resulting from siGFP treatment, as determined by quantitation by area under the curve (Figure 2F). Glucose tolerance tests (GTTs) also revealed that mice receiving both IRS1U6 and IRS2U6 were moderately glucose intolerant as compared with siGFP-treated controls, while there was no statistical difference when single viruses were injected, as determined by area under the curve (Figure 2, G and H).

Hypomorphic expression of IRS-1 and IRS-2 causes significant defects in insulin signaling in liver but not in other tissues. To better understand the molecular mechanisms of these physiological defects, we measured PY levels and PI3K activity in IRS immunoprecipitates from liver. As expected, IRS-1 PY levels in liver were substantially decreased in insulin-stimulated mice treated with IRS1U6. Moreover, IRS-1-associated PI3K activity was decreased by $35 \%$ in mice injected with IRS1U6, while mice treated with both RNAi adenoviruses showed a $45 \%$ decrease in IRS-1-associated PI3K activity (Figure 3A). In addition, IRS1U6 caused a modest $20 \%$ increase in IRS-2-associated PI3K activity along with a similar increase in IRS-2 expression (Figure 3B). These increases in IRS-2-associated PI3K activity and expression were both statistically significant. 


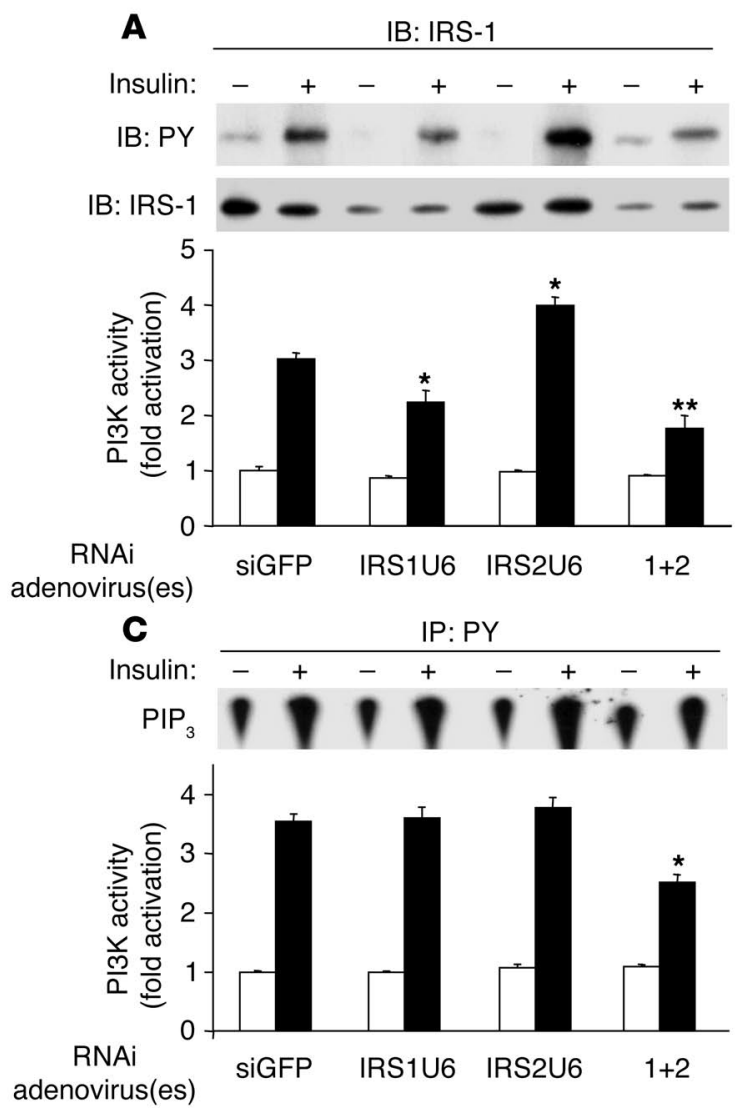

B

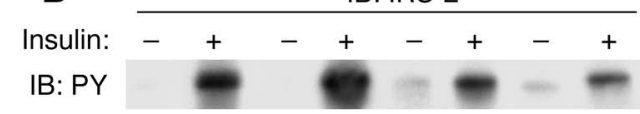

IB: IRS-2

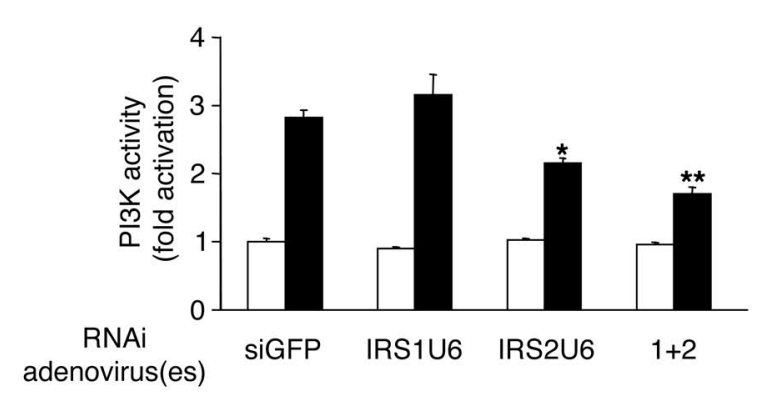

Figure 3

Hypomorphic expression of IRS-1 and IRS-2 causes defects in PI3K activation. (A) IRS-1 immunoprecipitates blotted with 4G10, an anti-PY antibody (top panel). PI3K activity was also measured from these IRS-1 immunoprecipitates (bottom panel, $n=4)$. (B) As in A, but with IRS-2 immunoprecipitates. (C) PI3K activity in PY-immunoprecipitates (bottom panel), and a representative phosphatidylinositol-( $3,4,5)$ trisphosphate $\left(\mathrm{PIP}_{3}\right)$ level (top). Bars represent mean \pm SEM $(n=4)$. ${ }^{\star} P<0.05$ vs. siGFP treatment; ${ }^{* \star} P<0.01$ vs. siGFP.

Similar patterns of tyrosine phosphorylation and PI3K activity were observed in IRS-2 immunoprecipitates. IRS2U6 treatment diminished insulin-stimulated IRS-2 phosphorylation when administered either alone or in combination with IRS1U6. A $25 \%$ decrease in IRS-2-associated PI3K activity was also observed in mice treated with only IRS2U6, and mice injected with both adenoviruses exhibited a $40 \%$ decrease in IRS-2-associated PI3K activity. Interestingly, when IRS-2 was knocked down acutely, IRS-1-associated PI3K activity increased by $30 \%$, without any increase in IRS-1 expression.

Despite these reductions in IRS tyrosine phosphorylation and IRS-specific changes in PI3K activity, there were no detectable differences in PY-associated PI3K activity when IRS-1 and IRS-2 expression were individually reduced (Figure 3C). Furthermore, insulin stimulation increased PY-associated PI3K activity by approximately 3.5 -fold in mice treated with siGFP, IRS1U6, or IRS2U6. However, when IRS-1 and IRS- 2 were both knocked down, there was a $40 \%$ decrease in PY-associated PI3K activity.

The changes in total PI3K activity correlated with the downstream activation of Akt. Thus, Akt phosphorylation and insulin-stimulated Akt activity were decreased only in mice with dual defects in IRS- 1 and IRS-2 expression (Figure 4, A and B). The administration of either IRS1U6 or IRS2U6 alone did not result in any detectable changes in Akt activation compared with control siGFP treatment.
Concomitant treatment with IRS1U6 and IRS2U6, on the other hand, caused a $50 \%$ decrease in serine 473 phosphorylation of Akt (Figure 4A) and a 60\% decrease in Akt kinase activity (Figure 4B) compared with control siGFP treatment.

These defects in Akt activation translated downstream to the decreased phosphorylation of Foxo1 but not of MAPK (Figure 4C). No reproducible decrease in Foxo1 phosphorylation was observed whether mice were treated with control siGFP or any single adenovirus, as determined by the average of 3 independent densitometric experiments, but dual knockdown resulted in a 50\% decrease in Foxo1 phosphorylation. On the other hand, MAPK phosphorylation surprisingly remained constant despite substantial knockdown of IRS-1 and IRS-2 protein levels.

Knockdown of hepatic IRS proteins causes indirect changes in adipocyte function. We also addressed the possibility that part of the insulin-resistant phenotype of the dual-knockdown mice may also be due to indirect effects in adipose tissue. Previous studies have shown that an adenovirus-mediated disruption of hepatic PI3K activity caused an indirect increase in epididymal fat pad mass with decreased PI3K activity in adipose tissue (30). IRS1U6 or IRS2U6, however, did not cause changes in epididymal fat pad size compared with control siGFP (Supplemental Figure 1A; supplemental material available online with this article; doi:10.1172/ JCI200523187DS1). Moreover, knockdown of IRS proteins in 
A RNA adenovirus(es): SiGFP IRS1U6 IRS2U6 $1+2$ Insulin $-+\overline{-+}+\overline{-+}$

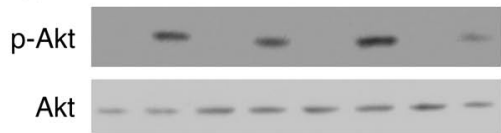

B

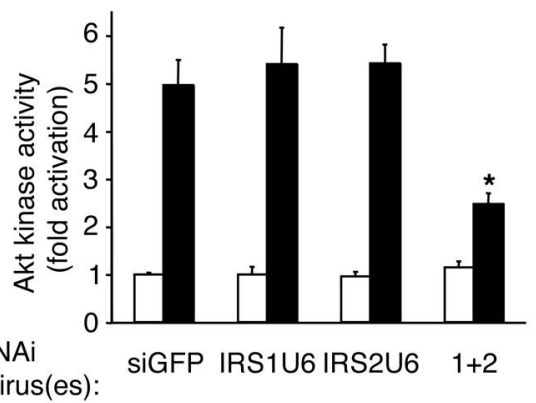

Figure 4

Significant defects in Akt activation and downstream targets of Akt in mice treated with IRS1U6 and IRS2U6. (A) Insulin-stimulated liver lysates from mice treated with RNAi adenovirus blotted with anti-phospho Akt (S473) antibody (top panel). Blots were then stripped and reprobed for total Akt (bottom panel). The blot is representative of 3 independent experiments. (B) Akt kinase activity was measured in Akt immunoprecipitates using Crosstide as a substrate (56). Bars represent mean $\pm \operatorname{SEM}(n=3)$. ${ }^{*} P<0.01$ dual treatment vs. siGFP. (C) Insulin-stimulated liver lysates from mice treated with RNAi adenovirus blotted with antibodies against phospho-Foxo1 (S256) or phopshoMAPK (T202/Y204). The blots for the phosphorylated proteins were then stripped and reprobed with antibodies for total Foxo1 or total MAPK. These Western blots are representative of 3 independent experiments.

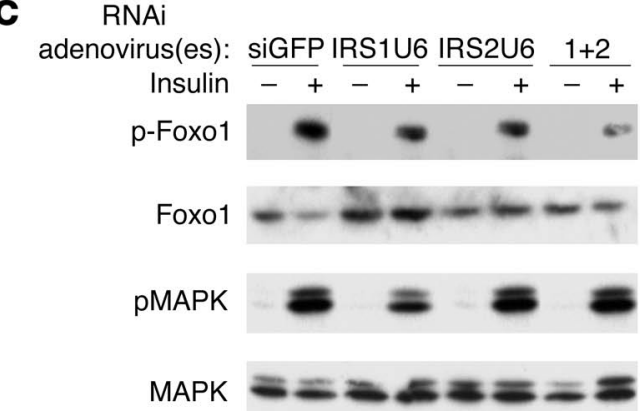

MAPK

genes were upregulated in livers of double-knockdown mice by more than 2-fold compared with controls. These results were confirmed by Northern blot analysis (data not shown). Interestingly, treatment with IRS1U6 alone also increased mRNA levels of PEPCK and G6Pase expression by $1.7-$ and 1.6-fold, respectively.

Glucokinase (Gck) plays a key role in regulating glucose homeostasis, and Gck gene mutations in humans cause maturity onset diabetes of the young, type 2 (MODY-2). The MODY phenotype may arise partly from dysregulated hepatic glucose disposal, since mice with a liver-specific ablation of glucokines exhibit severe hypergly-

liver did not alter tyrosine phosphorylation of IRS-1, IRS-2, and the insulin receptor, as well as the insulin-stimulated S473 phosphorylation of Akt (Supplemental Figure 2, A and B).

These findings, however, did not preclude the possibility that adipokine or FFA levels were altered in response to the acute knockdown of hepatic IRS-1 or IRS-2. The liver-specific knockdown of IRS- 1 or IRS- 2 did not result in a statistically significant change in serum adiponectin (Figure 5A). In contrast, serum leptin levels were significantly decreased in mice treated with IRS2U6 or receiving dual treatment, compared with siGFP treatment (Figure 5B). Decreasing hepatic IRS-1 expression exerted no effect on serum leptin levels. Interestingly, we detected a statistically significant increase in serum FFA levels in IRS-1- or IRS-2-knockdown mice and a 1.8-fold increase in dual-knockdown mice compared with controls.

Increased expression of gluconeogenic enzymes and decreased expression of glucokinase with IRS-1 knockdown. Since the phosphorylation of Foxo 1 by Akt inhibits its ability to activate gluconeogenesis (31), we reasoned that the decreased Foxo1 phosphorylation in the dual-knockdown mice may have caused increased expression of gluconeogenic genes. Using quantitative RT-PCR analysis, we measured the mRNA levels of several key gluconeogenic enzymes, phosphoenolpyruvate carboxykinase (PEPCK), glucose-6 phosphatase (G6Pase), and fructose-1,6-bisphosphatase (F-1,6BP) (Figure 6, A, B, and C, respectively). Indeed, all 3 gluconeogenic

\section{Figure 5}

Decreased leptin and increased FFA levels in IRS-knockdown mice. (A) Serum adiponectin levels of random-fed mice treated with RNAi adenovirus. (B) Serum leptin levels of random-fed mice treated with RNAi adenovirus. (C) Serum free fatty acid levels in random-fed knockdown mice. Bars represent mean $\pm \operatorname{SEM}(n=5)$. ${ }^{*} P<0.05$ vs. single treatment vs. siGFP treatment; ${ }^{* \star} P<0.01$ vs. siGFP treatment. cemia $(32,33)$. Thus, we directly measured Gck expression in livers of mice treated with RNAi adenovirus and found that Gck expression was tied closely with IRS-1 expression (Figure 6D). Treatment with IRS1U6 alone caused a 40\% drop in Gck expression, while dual treatment resulted in a $46 \%$ decrease in Gck levels. These RT-PCR results were also confirmed by Northern blot analysis (data not shown). Treatment with IRS2U6 resulted in a modest 25\% downregulation of Gck, which was not statistically significant.
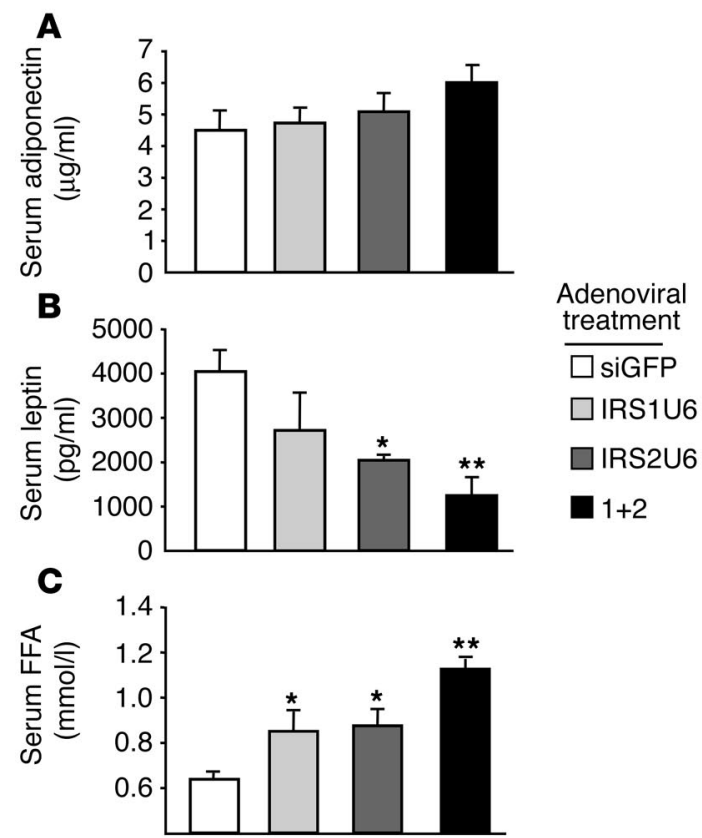


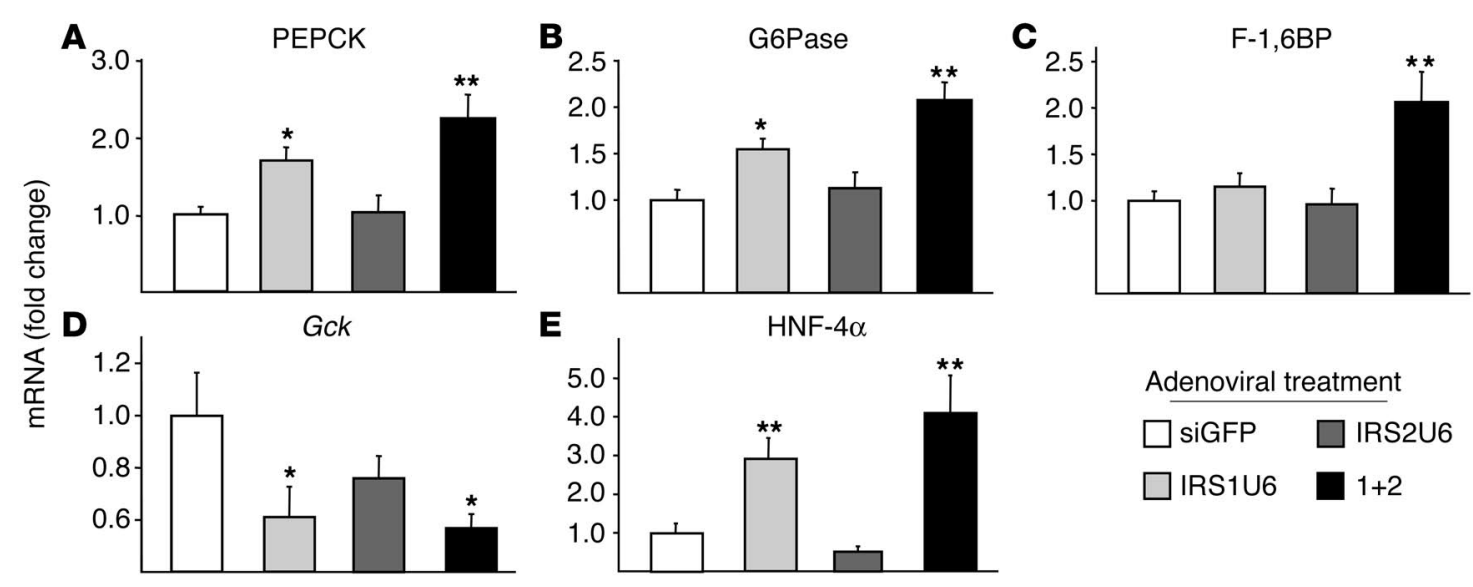

\section{Figure 6}

Downregulated IRS protein expression in liver results in changes in expression of gluconeogenic genes and Gck. The relative mRNA levels of indicated genes in the livers of fasting mice treated with RNAi adenovirus, as measured by quantitative RT-PCR: (A) PEPCK, (B) G6Pase, (C) F-1,6BP, (D) Gck, and (E) HNF-4 $\alpha$. Vertical axes represent the fold change in mRNA levels compared with siGFP treatment. The bars represent the fold change in expression of each gene relative to the mean expression in siGFP-treated controls \pm SEM $(n=8)$. ${ }^{\star} P<0.05$ vs. siGFP treatment; ${ }^{* *} P<0.01$ vs. siGFP.

We postulated that the association between decreased IRS-1 expression and increased gluconeogenic gene transcription might be explained by the altered expression of transcription factors upstream of PEPCK and G6Pase. Hepatic nuclear factor- $4 \alpha$ (HNF- $4 \alpha$ ) is a transcription factor that coordinates with the forkhead transcription factors to activate the gluconeogenic gene cassette (34), and, moreover, mutated versions of HNF- $4 \alpha$ are thought to cause MODY-1. RT-PCR analysis revealed a striking correlation between IRS-1 knockdown and the mRNA levels of HNF- $4 \alpha$, as the expression of HNF- $4 \alpha$ was increased approximately 3 -fold in the livers of mice treated with IRS1U6 alone and elevated by 4-fold in dual-treated mice (Figure 6E). Conversely, IRS2U6 resulted in a 50\% decrease in HNF- $4 \alpha$ mRNA expression, which was not statistically significant.

IRS-2 may specifically regulate lipid homeostasis by regulating lipogenic enzyme expression. The inappropriate activation of gluconeogen- esis in insulin-resistant states is often accompanied by defects in hepatic lipid regulation. We measured the expression of key lipogenic genes such as Srebf1c and fatty acid synthase (Fasn) in response to IRS-1 and IRS-2 knockdown by adenoviral shRNAs. Whereas IRS-1 knockdown alone did not have any effect on Srebf1c or Fasn expression, decreased hepatic IRS-2 expression alone was sufficient to induce a 1.6-fold upregulation of Srebf1c. Moreover, dual knockdown increased SREBP and Fasn expression by 3.2- and 2.6-fold, respectively (Figure 7, A and B).

Insulin may also regulate lipogenic gene transcription through pathways independent of the IRS proteins, most notably through liver X receptor (LXR) activation (35). Using quantitative RT-PCR analysis, we found that LXR $\alpha(N r 1 b 3)$ mRNA levels were unchanged after treatment with IRS1U6, IRS2U6, or both (Figure 7C). This result, however, does not preclude the possibility that LXR $\alpha$ activity
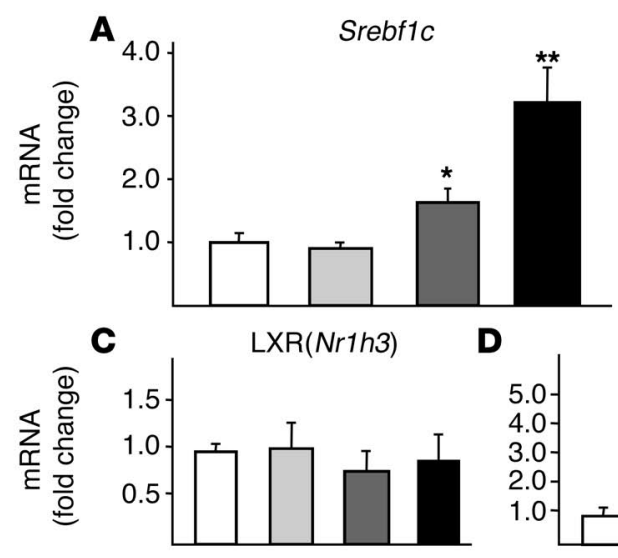

B
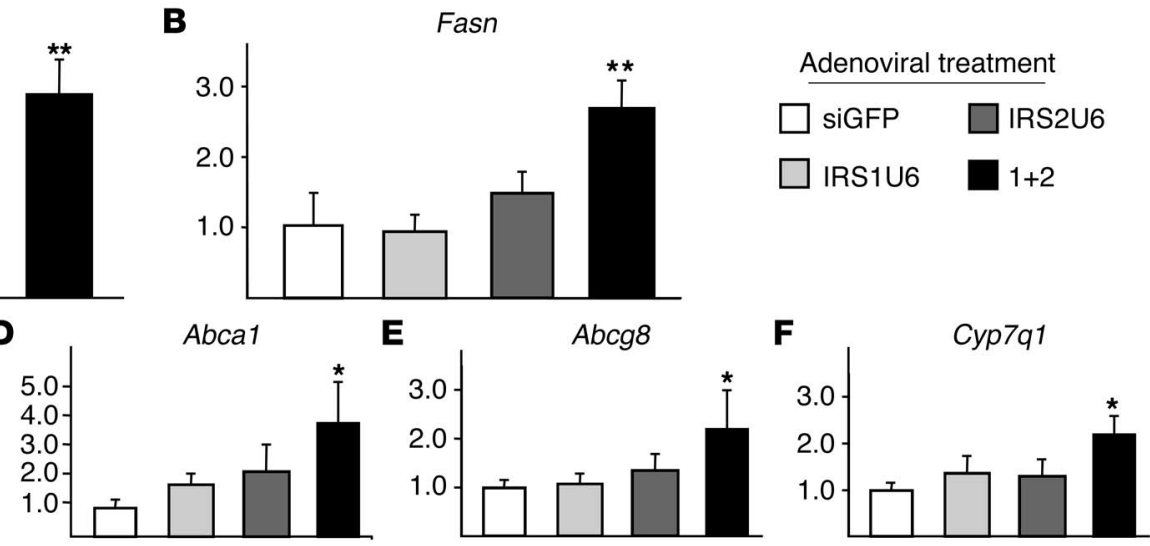

Figure 7

Decreased IRS-2 and hyperinsulinemia combine to cause increased lipogenic gene expression and activation of downstream LXR genes. The relative mRNA levels of lipogenic genes in the livers of fasting mice treated with RNAi adenovirus, as measured by RT-PCR: (A) SREBP-1C, (B) FAS, (C) LXR, (D) ABCA1, (E) ABCG8, and (F) CYP7A1. The vertical axes represent the fold change in mRNA levels compared with siGFP treatment. The bars represent the fold change in expression of each gene relative to the mean expression in siGFP-treated controls \pm SEM $(n=8)$. ${ }^{*} P<0.05$ vs. siGFP treatment; ${ }^{*} P<0.01$ vs. siGFP. 


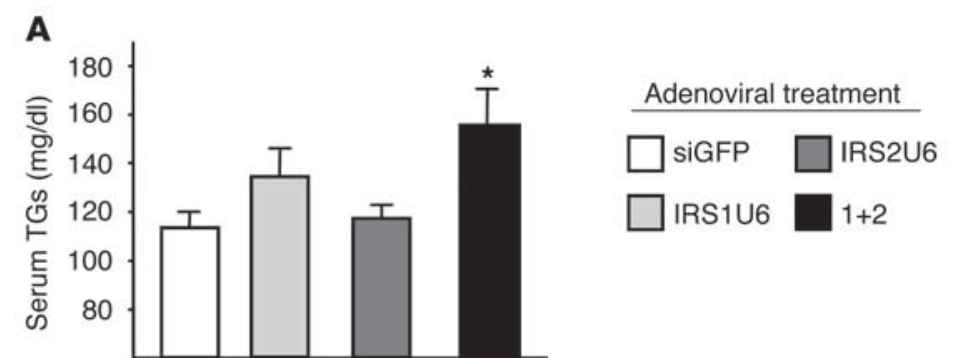

C
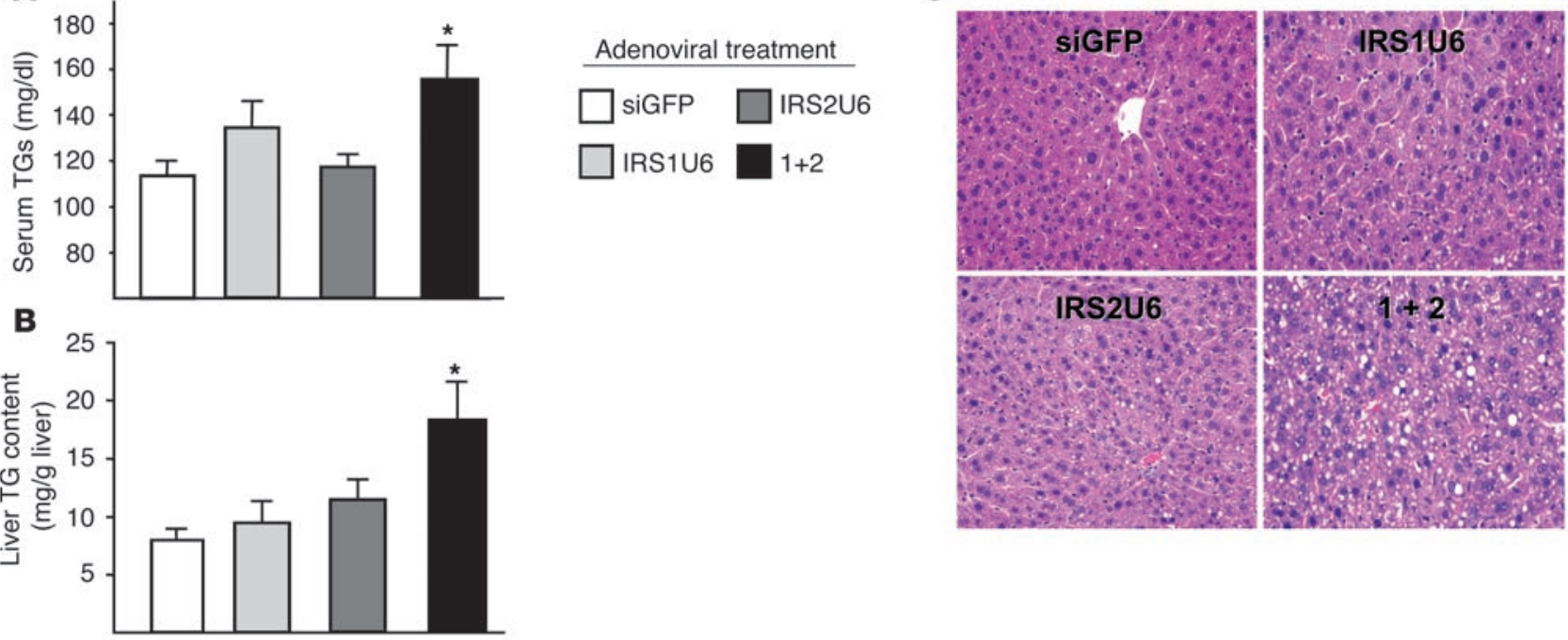

Figure 8

Dual knockdown of hepatic IRS-1 and IRS-2 causes dyslipidemia, increased liver TG content, and hepatic steatosis. (A) Dual knockdown causes increased serum TG levels. Each bar represents the mean serum TG content \pm SEM $(n=6)$. (B) Increased TG accumulation in the livers of mice treated with IRS1U6 and IRS2U6. Each bar represents the mean value of measured liver TG content per gram liver \pm SEM $(n=6)$. (C) H\&E staining of livers from fasted mice, 5 days after treatment with RNAi adenovirus. ${ }^{*} P<0.05$ vs. siGFP treatment. Magnification, $\times 400$.

may be increased. As a proxy for LXR activation, we measured the expression of downstream LXR $\alpha$ genes, such as ATP-binding cassette, subfamily A1 (Abca1; Figure 7D), Abcg8 (Figure 7E), and cytochrome P450, isoform 7A1 (Cyp7a1; Figure 7F). Indeed, dual knockdown of IRS-1 and IRS-2 resulted in a 2- to 3-fold increase in all of these LXR downstream genes compared with control siGFP treatment.

These increases in lipogenic gene expression correlated with increased triglyceride (TG) accumulation in the liver and serum. The simultaneous knockdown of IRS-1 and IRS-2 in liver resulted in elevated serum TG levels by 1.5 -fold compared with control siGFP adenovirus treatment (Figure 8A). In contrast, there was no statistical difference in serum TG levels between mice receiving single RNAi adenoviral treatments and controls. Liver TG content paralleled those of serum, with hepatic TG content elevated more than 3-fold by dual treatment compared with siGFP treatment, while the increases in liver TG content in mice treated with either IRS1U6 or IRS2U6 alone were more modest (Figure 8B). To assess whether this increased lipid in the serum and liver resulted in changes in liver morphology, we analyzed H\&E-stained sections of fixed liver from mice treated simultaneously with IRS1U6 and IRS2U6. Indeed, dual-treated mice exhibited a microvesicular hepatic steatosis that resembles fatty hepatic lesions observed in human patients with type 2 diabetes mellitus. Mice treated with only IRS2U6 showed mild fatty liver change, and IRS1U6-treated livers were similar to control siGFP-treated livers (Figure 8C).

\section{Discussion}

We have utilized a novel in vivo RNAi technique that uses an adenoviral vector to deliver shRNAs against IRS-1, IRS-2, or both, in the livers of WT mice. This adenovirus-mediated RNAi technique affords us spatio-temporal control over the expression of IRS-1 and IRS-2 in the liver and allows us to define their individual roles in hepatic insulin signaling and hepatic metabolism without the pleiotropic side effects that could occur in global-knockout animals.
We find that IRS- 1 and IRS-2 play complementary roles in the liver at the level of intermediate signaling, such as PI3K activation and Foxo 1 phosphorylation. Thus, $70-80 \%$ reductions of IRS- 1 or IRS-2 individually do not perturb total PI3K or Akt activity because a reciprocal increase in PI3K activity through the unaltered IRS antipode helps to maintain full PI3K signal. Similar compensatory mechanisms also occur in the livers of IRS-1-KO mice, where IRS-2 expression significantly increases to maintain total PI3K activity and Akt activation (15). In contradistinction, IRS-1 levels do not increase when IRS-2 is decreased by either RNAi or global genetic deletion (13). Thus, it is interesting to find that IRS-1-associated PI3K activity and IRS-1 tyrosine phosphorylation levels increase by $30 \%$ in our IRS-2-knockdown animals. IRS-2-KO mice do not exhibit this increase in IRS-1-associated PI3K activity in liver (36), which indicates that a lifelong loss of IRS-2 could produce different chronic adaptive changes in gene expression and signaling that would not occur in an acute loss of IRS-2, such as that induced by our shRNAs. This difference in compensatory responses may help to explain why IRS-2 downregulation by adenoviral shRNAs does not disrupt Akt activation, whereas IRS-2 KO results in an approximately 50\% decrease in hepatic Akt activation (36).

Interestingly, the knockdown of either IRS-1 or IRS-2 did not affect the insulin-stimulated phosphorylation of MAPK. The maintenance of MAPK signaling in the face of compromised upstream activation of the insulin receptor and IRS proteins has been previously observed in humans with diabetes (37). That substantial reductions in IRS proteins did not alter MAPK phosphorylation indicates that other docking proteins, such as Shc (38), may be interchangeable with the IRS proteins in maintaining MAPK signaling but not PI3K signaling.

In insulin-resistant and hyperinsulinemic states, there are usually simultaneous reductions in IRS-1 and IRS-2 in liver (8). Dual knockdown of IRS- 1 and IRS- 2 closely mimics this situation and has allowed us to explore how this molecular defect affects systemic 
metabolic homeostasis. The hypomorphic expression of both IRS-1 and IRS-2 at levels equivalent to the depleted levels in livers of obese diabetic animals (6) led to decreased Akt activation and Foxo1 phosphorylation in response to insulin, which would be expected to lead to inappropriate hepatic glucose production. Indeed, hyperglycemia and hyperinsulinemia were observed in dual-knockdown animals, along with components of the metabolic syndrome, such as insulin resistance, glucose intolerance, dyslipidemia, and hepatic steatosis. Thus, the data demonstrate that decreased hepatic IRS expression has a direct causative role in the pathobiology of diabetes.

The dual knockdown of IRS-1 and IRS-2 in liver also affected the function of other metabolic tissues. Although IRS tyrosine phosphorylation and protein levels in muscle and fat were not affected by the knockdown of IRS proteins in liver, serum leptin levels were dramatically decreased in mice receiving either IRS2U6 or dual treatment. The decrease in serum leptin in these mice cannot simply be explained by changes in adipose mass, since epididymal fat pad mass did not change. Consequently, this interesting effect of hepatic IRS-2 signaling must be a result of the complex systemic metabolic alterations that stem from hepatic insulin resistance. One possible explanation may be that the elevated serum lipids may adversely affect leptin release from white adipose tissue (39). Indeed, we did find that serum FFA levels were elevated approximately 1.5 -fold in the single knockdowns and almost 2-fold in the dual knockdown mice compared with controls. This increased FFA concentration in the serum may also partially explain the mild insulin resistance observed by ITT in the single-knockdown animals and the more severe insulin resistance observed in the dual-knockdown mice, since serum FFAs have been shown to cause insulin resistance in muscle and liver $(40,41)$.

Interestingly, although IRS- 1 and IRS- 2 work in a complementary fashion to maintain PI3K signaling, they have unique individual roles in regulating key genes in glucose and lipid homeostasis. The acute reduction of IRS-1 expression resulted in a significant increase in the mRNA abundance of the essential gluconeogenic enzymes PEPCK, G6Pase, and F-1,6BP. The increased expression of these genes may be a consequence of the striking upregulation of HNF- $4 \alpha$ mRNA that occurs with IRS- 1 downregulation. Our data also indicate that IRS- 1 is more prominent than IRS- 2 in the regulation of Gck expression, since IRS1U6, either alone or in combination with IRS2U6, caused an approximately 50\% decrease in Gck expression. A similar IRS-1-specific regulation of glucokinase mRNA levels has been observed in cultured hepatocytes (42), in which IRS-1 associated PI3K activity was specifically ablated by overexpression of an N-terminal IRS-1 fragment.

IRS-2, on the other hand, may be more essential to insulin's regulation of lipogenesis than IRS-1. The observed inverse relationship between IRS- 2 protein levels and SREBP transcription in our study has also been reported in IRS-2-KO mice (increased SREBP-1c) and in primary hepatocytes from SREBP-1-KO mice (increased IRS-2) $(5,19,43)$. A key difference in our in vivo model, however, is that the hypomorphic IRS-2 expression is limited to liver and is not complicated by effects of hyperinsulinemia, which also depletes IRS-2 (44), or the pleiotropic effects of a global lack of IRS-2 (19). Thus, decreased expression of IRS-2 in liver alone is sufficient to elevate levels of Srebf1c and can occur independently of the known positive regulatory effects of insulin on Srebf1c expression (45).

That decreased IRS-2 and hyperinsulinemia synergistically increase Srebf1c expression implies that insulin and IRS-2 affect Srebflc levels through different regulatory pathways. There are several distinct pathways that likely contribute to the dysregulated lipogenesis and hepatic steatosis observed in the dual-knockdown mice: the LXR pathway, the MAPK pathway, and leptin signaling. The increased LXR activity, as detected by increased expression of downstream LXR genes, in the dual-knockdown mice probably contributed significantly to the increased Srebf1c expression, since insulin-stimulated LXR activity has recently been shown to increase SREBP-1c transcription via a signaling-independent mechanism (35). The unfettered activation of MAPK in these RNAi knockdowns may have also contributed to the activation of lipogenesis, since MAPK has been known to be an important regulator of Srebf1c transcription (46). Last, the decreased serum leptin must have complemented the other noted defects to produce the hepatic dyslipidemia observed in the dual-knockdown mice, as leptin has been shown to have potent regulatory effect on hepatic steatosis (21).

Consequently, the dyslipidemia and hepatic steatosis that often accompany diabetes and the metabolic syndrome may be a result of several important changes in the liver caused by hyperinsulinemia: decreased IRS-2 expression, increased LXR activity, unaltered MAPK activity, and decreased leptin action. We cannot rule out the possibility, however, that the dyslipidemia of the dual knockdowns may have been caused by uncharacterized effects of the IRS proteins on fatty acid oxidation or TG clearance, rather than increased TG synthesis through upregulated SREBP-1c and FAS. The former scenario may be less likely, since the mRNA expression of these important fatty acid oxidation genes medium chain acyl-CoA dehydrogenase (Acadm) and carnitine palmitoyl transferase-1 (Cpt1a) were not changed by knockdown of IRS-1 or IRS-2 (Supplemental Figure 2, $\mathrm{B}$ and $\mathrm{C}$ ), though these qualitative data do not rule out the possibility of multiple or alternative defects in lipid homeostasis.

The signaling pathways by which IRS- 1 and IRS- 2 might differentially regulate glucose and lipid homeostasis are not clearly understood. Although their overall protein structure and signaling functions appear to be quite similar, IRS-1 and IRS-2 are known to differ in their cellular compartmentalization $(47,48)$ and activation kinetics (49). Furthermore, IRS-1 and IRS-2 may have intrinsically different abilities to bind various $\mathrm{SH} 2$-containing partners (50). Further studies must be performed to determine whether the changes in gene expression brought about by decreased IRS-1 or IRS-2 are due to differential partnering, compartmentalization, or some other feature of these proteins.

In summary, we have developed an adenovirus-mediated RNAi technique to knock down IRS-1 and IRS-2 in the livers of WT mice. The tissue and genetic specificity of this technique has enabled us to demonstrate that hepatic IRS-1 and IRS- 2 maintain metabolic homeostasis through their complementary roles in regulating insulin signaling and gene expression, with IRS-1 more closely linked to the regulation of gluconeogenesis and Gck expression and IRS-2 is more closely linked to the regulation of lipogenesis. Thus, the differential modulation of hepatic IRS expression and signaling may represent an important component of the molecular pathophysiology that underlies both type 2 diabetes mellitus and the metabolic syndrome. Moreover, adenovirus-mediated RNA interference represents a promising technique to further understand the complex network of hepatic gene function and how it relates to in vivo physiology.

\section{Methods}

Animals. Eight-week-old male C57BL/6 mice were purchased from Taconic Laboratories. All animals were kept on a 12-hour light-dark cycle and fed a standard rodent chow. All protocols for animal use and euthanasia were 
approved by the Animal Care Use Committee of the Joslin Diabetes Center and Harvard Medical School in accordance with NIH guidelines.

Quantitative RT-PCR analysis. Total RNA was isolated from mouse tissues using an RNeasy kit (QIAGEN). cDNA was prepared from $1 \mu \mathrm{g}$ of RNA using the Advantage RT-PCR kit (BD) with random hexamer primers, according to the manufacturer's instructions. The resulting cDNA was diluted 10 -fold, and a $5-\mu \mathrm{l}$ aliquot was used in a $20-\mu \mathrm{l}$ PCR reaction (SYBR Green; BD) containing primers at a concentration of $300 \mathrm{nM}$ each. PCR reactions were run in triplicate and quantitated using the $\mathrm{ABI}$ Prism 7700 Sequence Detection System (ABI). Cycle threshold $(\mathrm{Ct})$ values were normalized to TATA box binding protein expression, and results were expressed as a fold change of mRNA compared with siGFP control mice.

Metabolic studies. For GTT, blood samples were obtained at 0, 15, 30, 60, and 120 minutes after i.p. injection of $2 \mathrm{~g} / \mathrm{kg}$ dextrose. We performed insulin tolerance tests by injecting $1 \mathrm{U} / \mathrm{kg}$ insulin (Novolin; Novo Nordisk) i.p., with blood sampling at $0,15,30$, and 60 minutes after injection. Blood glucose values were determined using a One Touch II glucose monitor (LifeScan Inc.). Plasma insulin and leptin levels were measured by ELISA using mouse insulin or mouse leptin as a standard (Crystal Chem Inc.). Serum adiponectin levels were also measured by ELISA (Linco Research Inc.). TG levels in liver and serum from fasted animals were measured by a colorimetric enzyme assay using the GPO Trinder kit (Sigma-Aldrich). Serum FFA levels were measured using a colorimetric diagnostic kit (Wako Pure Chemical Industries Ltd.).

Generation of adenoviral shRNA constructs. The sequences of IRS-1 and IRS-2 mRNA were analyzed for compatible RNAi oligonucleotides using generally accepted design rules $(28,51)$. A 19 -nt sequence starting from nt 1752 of IRS-1 and a 21-nt sequence commencing from nt 109 from IRS-2 were synthesized as complementary antiparallel oligonucleotides with a loop sequence (ttcaagaga) and XhoI and XbaI compatible ends (52). The oligonucleotides were as follows for IRS1U6: tcgagGATGCACCACTTGGAACGTttcaag agaACGTTCCAAGTGGTGCATCttttt (forward) and ctagAAAAAGATGCACCACTTG GAACGTtctcttgaaACGTTCCAAGTGGTGCATCc (reverse). The oligonucleotides for IRS2U6 were: tcgagGAGTCAGCAGTTACCAAGTGttcaagagaCACTTGGTAACTGCTGACACA TCttttt (forward) and ctagAAAAAGATGTCAGCAGTTACCAAGTGtctcttgaaCACTTG GTAACTGCTGGACATC (reverse). The shRNA sequence against GFP for stable expression in vivo was described previously (23). The forward and reverse oligonucleotides were annealed and then ligated into a pBluescript (Stratagene) vector containing the human U6 promoter (IMG-800; Imgenex Corp.). The U6 construct was then placed into the SwaI site of the pAdex1CAwt cosmid cassette (Takara Bio Inc.). The recombinant adenoviruses, IRS1U6 and IRS2U6, were then formed by homologous recombination of the expression cosmid cassette and parental virus genome in HEK293 cells, as described previously (53).

Adenovirus-mediated RNA interference and in vivo insulin stimulation. Prior to in vivo use, all adenoviruses were purified on a cesium chloride gradient and dialyzed into PBS plus $10 \%$ glycerol. Eight-week-old male C57BL/6 mice were injected via tail vein with a adenoviral dose of $5 \times 10^{9}$ $\mathrm{PFU} / \mathrm{g}$ body weight, as described previously (54). For the combined IRS1U6 plus IRS2U6 treatments, a mixture containing a half dose of each virus was injected to keep the viral load constant between treat- ment groups. On the fifth day after injection, following an overnight fast, the mice were anesthetized with a ketamine/xylazine mixture, and injected with $5 \mathrm{U}$ of regular human insulin via the inferior vena cava. Five minutes after injection of the insulin bolus, liver, muscle and fat were removed and snap frozen in liquid nitrogen. Immunoprecipitation and immunoblot analysis of insulin signaling molecules were performed using tissue homogenates prepared in a tissue homogenization buffer that contained $25 \mathrm{mM}$ Tris- $\mathrm{HCl}$ ( $\mathrm{pH} 7.4$ ), $10 \mathrm{mM} \mathrm{Na}_{3} \mathrm{VO}_{4}, 100 \mathrm{mM} \mathrm{NaF}$, $50 \mathrm{mM} \mathrm{Na}_{4} \mathrm{P}_{2} \mathrm{O}_{7}, 10 \mathrm{mM}$ EGTA, $10 \mathrm{mM}$ EDTA, $2 \mathrm{mM}$ phenylmethylsulfonyl fluoride, and $1 \%$ Nonidet P40 supplemented with the Complete protease inhibitor cocktail (Roche Diagnostics) (16). All protein expression data were quantified by densitometry, and the reported knockdown represents the average of at least 3 independent experiments.

Liver enzymes. The serum levels of the liver enzymes AST and ALT were measured at Ani Lytics Inc. Since adenoviral treatment and RNAi both may trigger inflammation, we measured AST and ALT levels following adenoviral treatment and found that serum levels of these enzymes were not elevated beyond the normal limits for mouse physiology (data not shown).

Antibodies. Rabbit polyclonal anti-IRS-1 antibody (IRS-1), anti-IRS-2 antibody (IRS-2), and anti-IR antibody (IR) were generated as described previously (55). Rabbit polyclonal anti-Akt, anti-phospho Akt (S473), anti-phospho GSK3 (Ser9), anti-GSK3, anti-phospho Foxo1 (Ser256), anti-Foxo1, anti-phospho MAPK, and anti-MAPK antibodies were purchased from Cell Signaling Technology Inc. Goat polyclonal anti-Akt1/2 antibody (Akt) was purchased from Santa Cruz Biotechnology Inc. The monoclonal anti-PY antibody, 4G10, was purchased from Upstate Biotechnology Inc.

In vitro kinase assays. Tissue homogenates from liver, muscle or fat were extracted with tissue homogenization buffer and subjected to immunoprecipitation with IRS-1, IRS-2, or PY antibodies followed by PI3K assay as described previously (16). For Akt kinase activity, the enzyme was immunoprecipitated from lysates with a goat polyclonal Akt 1/2 antibody, and kinase activity measured using Crosstide (Upstate) as a substrate (56).

Statistics. Statistical significance between the multiple treatment groups was determined by ANOVA and Tukey's $t$ test. Statistical significance of ITT and GTT curves was determined by comparing differences in area under the curve, which was calculated by the trapezoid method (57).

\section{Acknowledgments}

We greatly appreciate the technical assistance of Laureen Mazzola and Will Wisdom. This work was supported by NIH grants DK33201 and DK55545, Joslin Diabetes and Endocrinology Research Center grant DK34834 (to C.R. Kahn), and a Medical Scientist Training Program grant (to C.M. Taniguchi).

Received for publication August 27, 2004, and accepted in revised form December 21, 2004.

Address correspondence to: C. Ronald Kahn, Joslin Diabetes Center, One Joslin Place, Boston, Massachusetts 02215, USA. Phone: (617) 732-2635; Fax: (617) 732-2593; E-mail: c.ronald. kahn@joslin.harvard.edu.

\footnotetext{
1. Tripathy, D., et al. 2004. Parallel manifestation of insulin resistance and beta cell decompensation is compatible with a common defect in Type 2 diabetes. Diabetologia. 47:782-793.

2. Kim, S.P., Ellmerer, M., Van Citters, G.W., and Bergman, R.N. 2003. Primacy of hepatic insulin resistance in the development of the metabolic syndrome induced by an isocaloric moderate-fat diet in the dog. Diabetes. 52:2453-2460.

3. Fisher, S.J., and Kahn, C.R. 2003. Insulin signaling
}

is required for insulin's direct and indirect action on hepatic glucose production. J. Clin. Invest. 111:463-468. doi:10.1172/JCI200316426.

4. Michael, M.D., et al. 2000. Loss of insulin signaling in hepatocytes leads to severe insulin resistance and progressive hepatic dysfunction. Mol. Cell. 6:87-97.

5. Shimomura, I., et al. 2000. Decreased IRS-2 and increased SREBP-1c lead to mixed insulin resistance and sensitivity in livers of lipodystrophic and ob/ob mice. Mol. Cell. 6:77-86.

6. Kerouz, N.J., Horsch, D., Pons, S., and Kahn, C.R. 1997. Differential regulation of insulin receptor substrates-1 and -2 (IRS-1 and IRS-2) and phosphatidylinositol 3-kinase isoforms in liver and muscle of the obese diabetic (ob/ob) mouse. J. Clin. Invest. 100:3164-3172.

7. Saad, M.J., et al. 1992. Regulation of insulin receptor substrate- 1 in liver and muscle of animal models of insulin resistance. J. Clin. Invest. 90:1839-1849. 
8. Anai, M., et al. 1998. Altered expression levels and impaired steps in the pathway to phosphatidylinositol 3-kinase activation via insulin receptor substrates 1 and 2 in Zucker fatty rats. Diabetes. 47:13-23.

9. Kim, Y.B., Peroni, O.D., Franke, T.F., and Kahn, B.B. 2000. Divergent regulation of Akt1 and Akt2 isoforms in insulin target tissues of obese Zucker rats. Diabetes. 49:847-856

10. Aytug, S., Reich, D., Sapiro, L.E., Bernstein, D., and Begum, N. 2003. Impaired IRS-1/PI3-kinase signaling in patients with HCV: a mechanism for increased prevalence of type 2 diabetes. Hepatology. 38:1384-1392.

11. Sun, Y., et al. 2002. Phosphoenolpyruvate carboxykinase overexpression selectively attenuates insulin signaling and hepatic insulin sensitivity in transgenic mice. J. Biol. Chem. 277:23301-23307.

12. Araki, E., et al. 1994. Alternative pathway of insulin signaling in targeted disruption of the IRS-1 gene. Nature. 372:186-190.

13. Withers, D.J., et al. 1998. Disruption of IRS-2 causes type 2 diabetes in mice. Nature. 391:900-904.

14. Withers, D.J., et al. 1999. Irs-2 coordinates Igf-1 receptor-mediated beta-cell development and peripheral insulin signalling. Nat. Genet. 23:32-40.

15. Yamauchi, T., et al. 1996. Insulin signalling and insulin actions in the muscles and livers of insulin-resistant, insulin receptor substrate 1-deficient mice. Mol. Cell. Biol. 16:3074-3084.

16. Ueki, K., et al. 2000. Restored insulin-sensitivity in IRS-1-deficient mice treated by adenovirus-mediated gene therapy. J. Clin. Invest. 105:1437-1445.

17. Previs, S.F., Withers, D.J., Ren, J.M., White, M.F., and Shulman, G.I. 2000. Contrasting effects of IRS-1 versus IRS-2 gene disruption on carbohydrate and lipid metabolism in vivo. J. Biol. Chem. 275:38990-38994.

18. Kubota, N., et al. 2000. Disruption of insulin receptor substrate 2 causes type 2 diabetes because of liver insulin resistance and lack of compensatory beta-cell hyperplasia. Diabetes. 49:1880-1889.

19. Tobe, K., et al. 2001. Increased expression of the sterol regulatory element-binding protein-1 gene in insulin receptor substrate-2(-/-) mouse liver. J. Biol. Chem. 276:38337-38340.

20. Suzuki, R., et al. 2004. Both insulin signaling defects in the liver and obesity contribute to insulin resistance and cause diabetes in irs2-/- mice. J. Biol. Chem. 279:25039-25049.

21. Asilmaz, E., et al. 2004. Site and mechanism of leptin action in a rodent form of congenital lipodystrophy. J. Clin. Invest. 113:414-424. doi:10.1172/ JCI200419511.

22. Shinagawa, T., and Ishii, S. 2003. Generation of Ski-knockdown mice by expressing a long doublestrand RNA from an RNA polymerase II promoter. Genes Dev. 17:1340-1345.

23. Rubinson, D.A., et al. 2003. A lentivirus-based system to functionally silence genes in primary mammalian cells, stem cells and transgenic mice by RNA interference. Nat. Genet. 33:401-406.

24. Kasim, V., Miyagishi, M., and Taira, K. 2004. Control of siRNA expression using the Cre-loxP recombination system. Nucleic Acids Res. 32:e66.

25. Ventura, A., et al. 2004. Cre-lox-regulated condi- tional RNA interference from transgenes. Proc. Natl. Acad. Sci. U. S. A. 101:10380-10385.

26. Tiscornia, G., Tergaonkar, V., Galimi, F., and Verma, I.M. 2004. CRE recombinase-inducible RNA interference mediated by lentiviral vectors. Proc. Natl. Acad. Sci. U. S. A. 101:7347-7351.

27. Koo, S.H., et al. 2004. PGC-1 promotes insulin resistance in liver through PPAR-alpha-dependent induction of TRB-3. Nat. Med. 10:530-534.

28. Hannon, G.J. 2002. RNA interference. Nature. 418:244-251.

29. Lee, Y.S., et al. 2004. Distinct roles for Drosophila Dicer-1 and Dicer-2 in the siRNA/miRNA silencing pathways. Cell. 117:69-81.

30. Miyake, K., et al. 2002. Hyperinsulinemia, glucose intolerance, and dyslipidemia induced by acute inhibition of phosphoinositide 3-kinase signaling in the liver. J. Clin. Invest. 110:1483-1491. doi:10.1172/JCI200215880.

31. Puigserver, P., et al. 2003. Insulin-regulated hepatic gluconeogenesis through FOXO1-PGC-1alpha interaction. Nature. 423:550-555.

32. Froguel, P., et al. 1993. Familial hyperglycemia due to mutations in glucokinase. Definition of a subtype of diabetes mellitus. N. Engl. J. Med. 328:697-702.

33. Postic, C., et al. 1999. Dual roles for glucokinase in glucose homeostasis as determined by liver and pancreatic beta cell-specific gene knock-outs using Cre recombinase. J. Biol. Chem. 274:305-315.

34. Rhee, J., et al. 2003. Regulation of hepatic fasting response by PPARgamma coactivator-1alpha (PGC-1): requirement for hepatocyte nuclear factor 4alpha in gluconeogenesis. Proc. Natl. Acad. Sci. U. S. A. 100:4012-4017.

35. Chen, G., Liang, G., Ou, J., Goldstein, J.L., and Brown, M.S. 2004. Central role for liver X receptor in insulin-mediated activation of Srebp-1c transcription and stimulation of fatty acid synthesis in liver. Proc. Natl. Acad. Sci. U. S. A 101:11245-11250.

36. Valverde, A.M., et al. 2003. Molecular mechanisms of insulin resistance in IRS-2-deficient hepatocytes. Diabetes. 52:2239-2248.

37. Cusi, K., et al. 2000. Insulin resistance differentially affects the PI 3-kinase- and MAP kinase-mediated signaling in human muscle. J. Clin. Invest. 105:311-320.

38. Pelicci, G., et al. 1992. A novel transforming protein (SHC) with an SH2 domain is implicated in mitogenic signal transduction. Cell. 70:93-104.

39. Cammisotto, P.G., Gelinas, Y., Deshaies, Y., and Bukowiecki, L.J. 2003. Regulation of leptin secretion from white adipocytes by free fatty acids. Am. J. Physiol. Endocrinol. Metab. 285:E521-E526.

40. Dresner, A., et al. 1999. Effects of free fatty acids on glucose transport and IRS-1-associated phosphatidylinositol 3-kinase activity. J. Clin. Invest. 103:253-259.

41. Bergman, R.N., and Ader, M. 2000. Free fatty acids and pathogenesis of type 2 diabetes mellitus. Trends Endocrinol. Metab. 11:351-356.

42. Matsumoto, M., et al. 2002. Role of the insulin receptor substrate 1 and phosphatidylinositol 3-kinase signaling pathway in insulin-induced expression of sterol regulatory element binding protein $1 \mathrm{c}$ and glucokinase genes in rat hepatocytes. Diabetes. 51:1672-1680.

43. Ide, T., et al. 2004. SREBPs suppress IRS-2-medi- ated insulin signalling in the liver. Nat. Cell Biol. 6:351-357.

44. Hirashima, Y., et al. 2003. Insulin down-regulates insulin receptor substrate-2 expression through the phosphatidylinositol 3-kinase/Akt pathway. J. Endocrinol. 179:253-266.

45. Shimomura, I., et al. 1999. Insulin selectively increases SREBP-1c mRNA in the livers of rats with streptozotocin-induced diabetes. Proc. Natl. Acad. Sci. U. S. A. 96:13656-13661.

46. Kotzka, J., et al. 1998. ADD1/SREBP-1c mediates insulin-induced gene expression linked to the MAP kinase pathway. Biochem. Biophys. Res. Commun. 249:375-379.

47. Inoue, G., Cheatham, B., Emkey, R., and Kahn, C.R. 1998. Dynamics of insulin signaling in 3T3-L1 adipocytes. Differential compartmentalization and trafficking of insulin receptor substrate (IRS)-1 and IRS-2. J. Biol. Chem. 273:11548-11555.

48. Sun, H., et al. 2003. Insulin-like growth factor I receptor signaling and nuclear translocation of insulin receptor substrates 1 and 2. Mol. Endocrinol. 17:472-486.

49. Ogihara, T., et al. 1997. Insulin receptor substrate (IRS)-2 is dephosphorylated more rapidly than IRS-1 via its association with phosphatidylinositol 3-kinase in skeletal muscle cells. J. Biol. Chem. 272:12868-12873.

50. Sun, X.J., et al. 1997. The IRS-2 gene on murine chromosome 8 encodes a unique signaling adapter for insulin and cytokine action. Mol. Endocrinol. 11:251-262.

51. Reynolds, A., et al. 2004. Rational siRNA design for RNA interference. Nat. Biotechnol. 22:326-330.

52. Sui, G., et al. 2002. A DNA vector-based RNAi technology to suppress gene expression in mammalian cells. Proc. Natl. Acad. Sci. U. S. A. 99:5515-5520.

53. Ueki, K., Kondo, T., and Kahn, C.R. 2004. Suppressor of cytokine signaling 1 (SOCS-1) and SOCS-3 cause insulin resistance through inhibition of tyrosine phosphorylation of insulin receptor substrate proteins by discrete mechanisms. Mol. Cell. Biol. 24:5434-5446.

54. Ueki, K., Kondo, T., Tseng, Y.H., and Kahn, C.R. 2004. Central role of suppressors of cytokine signaling proteins in hepatic steatosis, insulin resistance, and the metabolic syndrome in the mouse. Proc. Natl. Acad. Sci. U. S. A. 101:10422-10427.

55. Bruning, J.C., et al. 1997. Development of a novel polygenic model of NIDDM in mice heterozygous for IR and IRS-1 null alleles. Cell. 88:561-572.

56. Ueki, K., et al. 1998. Potential role of protein kinase $\mathrm{B}$ in insulin-induced glucose transport, glycogen synthesis, and protein synthesis. J. Biol. Chem. 273:5315-5322.

57. Tran, T.T., et al. 2003. Direct measure of insulin sensitivity with the hyperinsulinemic-euglycemic clamp and surrogate measures of insulin sensitivity with the oral glucose tolerance test: correlations with aberrant crypt foci promotion in rats. Cancer Epidemiol. Biomarkers Prev. 12:47-56.

58. Goren, H.J., Kulkarni, R.N., and Kahn, C.R. 2004. Glucose homeostasis and tissue transcript content of insulin signaling intermediates in four inbred strains of mice: C57BL/6, C57BLKS/6, DBA/2, and 129X1. Endocrinology. 145:3307-3323. 